\title{
PREPARAÇÃO E CARACTERIZAÇÃO DE ADSORVENTES PARA A REMOÇÃO DE SURFACTANTES ANIÔNICOS EM ÁGUAS RESIDUÁRIAS
}

\author{
G. L. COLPANI ${ }^{1}$ e R. F. P. M. MOREIRA ${ }^{1}$ \\ ${ }^{1}$ Universidade Federal de Santa Catarina - Centro Tecnológico - Departamento de Engenharia \\ Química e Engenharia de Alimentos \\ E-mail para contato: g_colpani@yahoo.com.br
}

\begin{abstract}
RESUMO - A preparação de adsorventes economicamente viáveis a partir de precursores oriundos de resíduos industriais tem sido avaliada no intuito de otimizar o processo de adsorção. Desta forma, os resíduos da avicultura foram utilizados para produção de carvões ativados com ácido fosfórico, analisando-se a influência da temperatura de carbonização, concentração de ácido fosfórico e tempo de ativação. As condições de ativação de 1 hora a $600{ }^{\circ} \mathrm{C}$, utilizando-se a razão mássica $\mathrm{H}_{3} \mathrm{PO}_{4}: \mathrm{H}_{2} \mathrm{O}$ igual 1:1 resultaram em carvões com área superficial BET de $664 \mathrm{~m}^{2} \cdot \mathrm{g}^{-1}$ e volume de mesoporos de $0,868 \mathrm{~cm}^{3} \cdot \mathrm{g}^{-1}$, os quais foram utilizados para a remoção de surfactantes aniônicos em efluentes líquidos. $\mathrm{O}$ modelo cinético de pseudossegunda ordem descreveu mais adequadamente a cinética de adsorção experimental, com $\mathrm{k}_{2}=$ $0,019 \mathrm{~g} \cdot \mathrm{mg}^{-1} \cdot \mathrm{min}^{-1} \mathrm{e} \mathrm{q}_{\mathrm{e}}=0,575 \mathrm{mg} \cdot \mathrm{g}^{-1}$. A isoterma de adsorção ajustou-se melhor ao modelo de Freundlich com $\mathrm{K}_{\mathrm{F}}=0,519\left(\left(\mathrm{mg} \cdot \mathrm{g}^{-1}\right) \cdot\left(\mathrm{L} \cdot \mathrm{mg}^{-1}\right)^{1 / \mathrm{n}}\right)$, sendo definida como uma adsorção favorável.
\end{abstract}

\section{INTRODUÇÃO}

O desenvolvimento econômico tem impulsionado o crescimento do setor industrial e tecnológico, consumindo recursos naturais e comprometendo os sistemas hídricos devido à introdução de poluentes como surfactantes, metais pesados e compostos organoclorados, o que reduz a disponibilidade de água potável e aumenta os custos no processo de tratamento (Pinheiro Filho, 2008).

Os surfactantes em águas de abastecimento e efluentes são um problema ambiental, devido a formação de espumas e consequente redução na transferência de oxigênio à biota aquática, além de concentrações acima de $1 \mathrm{mg} . \mathrm{L}^{-1}$ causarem efeitos tóxicos a mesma. A presença destes compostos em estações de tratamentos de efluentes causa um decréscimo na eficiência da etapa biológica, pelo efeito tóxico e formação de espumas e também nos processos físico-químicos, pois reduz a capacidade de floculação e consequentemente a sedimentação de sólidos presentes nos efluentes (Aboulhassan et al., 2006; Mungray e Kumar, 2008; Taffarel e Rúbio, 2010). Foi também reportado que, em estações de tratamento anaeróbio, os surfactantes são inibidores severos da digestão, sendo removidos somente com baixa eficiência (Gavalla, 2002). 


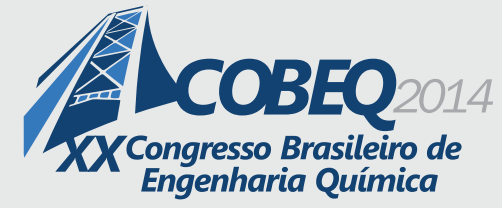

Dentre os processos para tratamento de águas e efluentes que têm sido investigados para reduzir estes elementos de forma eficaz e economicamente viável, a adsorção tem despertado grande interesse por remover eficientemente surfactantes presentes em corpos d'água devido à alta seletividade e estrutura porosa bem desenvolvida, permitindo a separação com um custo energético baixo. Os adsorventes mais comumente empregados no tratamento de efluentes líquidos são os carvões ativados, as zeólitas e algumas argilas. Carvões ativados podem ser preparados a partir de diferentes precursores, dentre os quais podem ser citados os resíduos agroindustriais, tais como a cama de aviário, rica em material carbonáceo.

O presente trabalho irá avaliar a capacidade de adsorção de surfactantes aniônicos a partir do emprego de um carvão ativado quimicamente com ácido fosfórico, na forma de pó finamente dividido, utilizando cama de aviário como precursor, para remoção de tensoativos em efluentes líquidos de agroindústria.

\section{MATERIAIS E MÉTODOS}

\subsection{Material Adsorvente}

O precursor do carvão ativado foi coletado de uma cama de aviário de frango, constituída principalmente por pinus, da região de Guatambú, estado de Santa Catarina. O material foi separado em peneira de 14 mesh Tyler - diâmetro de $1,18 \mathrm{~mm}$ - para remover sujidades e cavacos com granulometria elevada, sendo posteriormente lavado com ácido sulfúrico para remoção de compostos orgânicos e inorgânicos solúveis e amônia presente nas fezes depositadas sobre a cama de aviário, mantendo-se somente o material celulósico necessário. $\mathrm{O}$ procedimento de lavagem com ácido foi realizado empregando-se as metodologias propostas por Serrano et al. (2005) e Lima et al. (2008), sendo algumas etapas alteradas na tentativa de otimizar o processo.

\subsection{Ativação e Carbonização das Amostras}

As amostras foram ativadas com ácido fosfórico $\left(\mathrm{H}_{3} \mathrm{PO}_{4}\right) 85 \%$ e água destilada totalizando uma solução de $150 \mathrm{ml}$, sendo o processo conduzido a $85^{\circ} \mathrm{C}$ e $120 \mathrm{rpm}$ para todas as amostras, alterando-se nesta etapa somente o tempo de ativação e as razões $\mathrm{H}_{3} \mathrm{PO}_{4}: \mathrm{H}_{2} \mathrm{O}$, conforme Tabela 1 , sendo que após ativação, as amostras foram mantidas em estufa por 24 horas à temperatura de $85^{\circ} \mathrm{C}$.

Tabela 1 - Condições de ativação química e carbonização

\begin{tabular}{|c|c|c|c|c|c|c|c|c|c|}
\hline Amostra & CA01 & CA01 & CA01 & CA01 & CA01 & CA01 & CA01 & CA01 & CA01 \\
\hline Relação $\mathbf{H}_{\mathbf{2}} \mathbf{O}: \mathbf{H}_{\mathbf{3}} \mathbf{P O}_{\mathbf{4}}$ & $1: 1$ & $1: 1$ & $1: 1$ & $1: 1$ & $1: 2$ & $1: 3$ & $1: 3$ & $1: 3$ & $1: 3$ \\
\hline Tempo de Ativação (h) & 1 & 1 & 3 & 3 & 2 & 1 & 1 & 3 & 3 \\
\hline Temperatura de Carbonização $\left({ }^{\circ} \mathbf{C}\right)$ & 400 & 600 & 400 & 600 & 500 & 400 & 600 & 400 & 600 \\
\hline
\end{tabular}




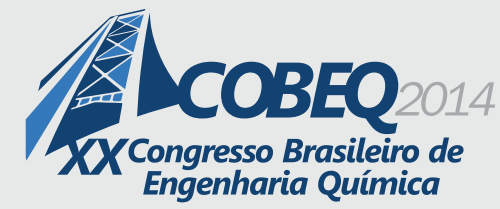

As amostras foram carbonizadas por duas horas em mufla sob atmosfera inerte de nitrogênio, com fluxo de $20 \mathrm{~mL} \cdot \mathrm{min}^{-1}$. O material foi lavado com água destilada até se estabilizar o pH. Posteriormente, a amostra foi seca em estufa à $110^{\circ} \mathrm{C}$ por 24 horas.

\subsection{Cinética de Adsorção}

As cinéticas de adsorção foram realizadas empregando-se um grama de carvão em $500 \mathrm{ml}$ de efluente bruto. $\mathrm{O}$ sistema foi mantido sob agitação constante e a temperatura de $20{ }^{\circ} \mathrm{C}$ através de banho termostático. As análises para determinação da concentração de surfactantes foram realizadas através de kit específico, denominado Spectro Kit - Reação Colorimétrica para Espectrofotômetro, o qual é adaptado do Standard Methods for the Examination of Water and Wastewater (APHA, 2005) e da NBR 10738 (ABNT, 1989), sendo coletadas alíquotas de $10 \mathrm{~mL}$ a cada 30 minutos e no equilíbrio, as quais foram avaliadas em espectrofotômetro no comprimento de onda de $652 \mathrm{~nm}$. O adsorvente preparado a partir de cama de aviário foi comparado ao carvão comercial fornecido pela empresa Norit Activated Carbon, denominado Norit CA 1

\subsection{Isoterma de Adsorção}

O procedimento para determinação das isotermas de adsorção consistiu em adicionar um grama de carvão em béqueres encamisados que possuíam volumes de 20, 40, 60, 80, 100 e 120 $\mathrm{mL}$ de efluente bruto. A concentração inicial de surfactantes aniônicos presentes no efluente foi ajustada para $4 \mathrm{mg} . \mathrm{L}^{-1}$ em todos os sistemas operacionais de adsorção, sendo o sistema mantido à temperatura de $20{ }^{\circ} \mathrm{C}$ através de banho termostático e sob agitação constante, durante 24 horas. Ao término deste período, foram coletadas alíquotas em triplicata, sendo suas concentrações de tensoativos aniônicos determinadas pela reação com azul de metileno.

\subsection{Caracterização de Carvões Ativados}

Os materiais adsorventes foram caracterizados através da determinação da área superficial pela metodologia da isoterma BET de adsorção de nitrogênio a $77 \mathrm{~K}$, através do equipamento Quantachrome NovaWin, presente no Laboratório de Processos de Separação e Sistemas Particulados (LPS) da Universidade Estadual de Maringá. As análises de microscopia de varredura eletrônica (MEV) foram realizadas utilizando-se o equipamento JEOL JSM-6390LV, do Laboratório Central de Microscopia Eletrônica (LCME) da Universidade Federal de Santa Catarina, para determinação morfológica das amostras de carvão ativado.

\section{RESULTADOS E DISCUSSÕES}

\subsection{Caracterização de Carvões Ativados}

Microscopia de varredura eletrônica: A análise das microscopias de varredura eletrônica permitiu a observação de um aumento da porosidade após processo de ativação em relação à matéria-prima, conforme observado na Figura 2. 

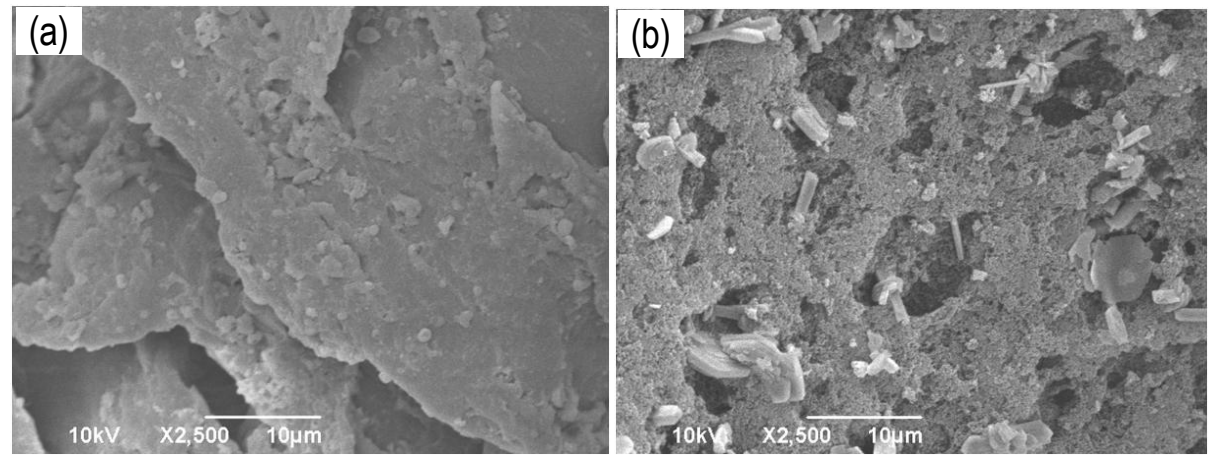

Figura 2 - Microscopia de varredura eletrônica. (a) Matéria-prima e (b) carvão ativado.

O aumento da porosidade ocorreu devido à agressividade do ácido fosfórico e também pela ação deste que atua como um catalisador para promover a ruptura de ligações, formando ésteres fosfóricos pela ligação do agente ativador com os grupos hidroxila da lignina. Isto provoca o afastamento das cadeias, gerando uma expansão da estrutura e redistribuição dos carbonos remanescentes, ocorrendo uma posterior polimerização destes grupos fosfatos sob aumento de temperatura, originando poros mais amplos. Após a remoção do ácido a matriz apresentará um estado expandido com uma estrutura de poros acessíveis (Nahil e Williams, 2012).

Análise de área superficial e volume e tamanho dos poros: As áreas superficiais foram analisadas pelo método BET, enquanto os volumes de poros e seus tamanhos foram avaliados durante a geração das isotermas pelos métodos BJH, DR (Dubinin e Radushkevich) e HK, sendo os resultados apresentados na Tabela 2.

Tabela 2 - Área superficial do adsorvente, volume e largura de poros

\begin{tabular}{|c|c|c|c|c|c|c|}
\hline Amostra & $\mathbf{S}_{\mathbf{B E T}}\left(\mathbf{m}^{\mathbf{2}} \cdot \mathbf{g}^{-\mathbf{1}}\right)$ & $\mathbf{V}_{\text {meso }}\left(\mathbf{c m}^{\mathbf{3}} \cdot \mathbf{g}^{\mathbf{- 1}}\right)$ & $\mathbf{V}_{\text {micro }}\left(\mathbf{c m}^{\mathbf{3}} \cdot \mathbf{g}^{\mathbf{- 1}}\right)$ & $\mathbf{S}_{\text {meso }}\left(\mathbf{m}^{\left.\mathbf{2} \cdot \mathbf{g}^{-\mathbf{1}}\right)}\right.$ & $\mathbf{S}_{\text {micro }}\left(\mathbf{m}^{\mathbf{2}} \cdot \mathbf{g}^{-\mathbf{1}}\right)$ & $\mathbf{D}_{\text {poros }}(\AA)$ \\
\hline MP & 272,4 & 0,264 & 0,126 & 167,8 & - & 21,30 \\
\hline CA01 & 428,2 & 0,076 & 0,188 & 60,29 & 146,5 & 12,81 \\
\hline CA02 & 663,9 & 0,868 & 0,323 & 373,1 & 15,35 & 31,65 \\
\hline CA03 & 415,1 & 0,135 & 0,206 & 106,1 & 91,72 & 14,64 \\
\hline CA04 & 573,5 & 0,624 & 0,283 & 285,7 & 15,03 & 27,87 \\
\hline CA05 & 302,4 & 0,186 & 0,151 & 105,7 & 27,08 & 19,79 \\
\hline CA07 & 522,5 & 0,331 & 0,317 & 206,7 & 46,23 & 20,79 \\
\hline CA08 & 276,4 & 0,237 & 0,142 & 151,1 & 154,6 & 22,42 \\
\hline CA09 & 406,5 & 0,182 & 0,212 & 127,3 & 210,0 & 17,12 \\
\hline
\end{tabular}




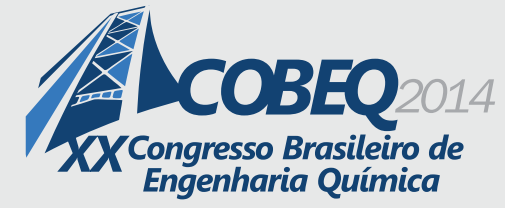

Os dados experimentais obtidos pela análise BET demonstram que o adsorvente com maior área superficial, volume de mesoporos e diâmetro de poros é a amostra CA02, a qual foi ativada em uma razão 1:1 $\left(\mathrm{H}_{3} \mathrm{PO}_{4}: \mathrm{H}_{2} \mathrm{O}\right)$ durante 1 hora e carbonizada a $600{ }^{\circ} \mathrm{C}$. Além disso, os resultados obtidos apresentam uma influência significativa da temperatura e impregnação no volume de mesoporos, sendo que o aumento da temperatura para razões de impregnação constantes aumenta tal volume. No entanto, quando se observam nas mesmas temperaturas de carbonização de 600 ${ }^{\circ} \mathrm{C}$ um aumento na concentração de ácido fosfórico, ocorre uma redução no volume de mesoporos. Tal relação ocorre possivelmente devido degradação da estrutura, convertendo mesoporos a macroporos.

O diâmetro dos poros conduz a uma avaliação prévia de que a amostra CA02 é a mais apropriada à adsorção de tensoativos aniônicos, pois o diâmetro médio dos poros é de 31,65 A, sendo definido pela IUPAC como mesoporos.

\subsection{Cinética de Adsorção}

As cinéticas de adsorção foram desenvolvidas no intuito de compreender o mecanismo que rege este processo, sendo os resultados ajustados pelos modelos de cinética de pseudo-primeira ordem, pseudossegunda ordem e difusão intrapartícula. A avaliação quantitativa dos modelos foi realizada pela comparação dos coeficientes de determinação $\left(\mathrm{R}^{2}\right)$ e pelos valores de $\mathrm{q}_{\mathrm{e}}$ calculados - $\mathrm{q}_{\mathrm{e}}$ (calc.) - versus os resultados de $\mathrm{q}_{\mathrm{e}}$ obtidos experimentalmente $-\mathrm{q}_{\mathrm{e}}$ (exp.).

O mecanismo de adsorção de pseudossegunda ordem foi o que melhor se ajustou aos dados experimentais, demonstrado na Figura 3, por apresentar os menores desvios entre a quantidade adsorvida calculada e experimental no equilíbrio e os maiores coeficientes de determinação, os quais são apresentados na Tabela 3. Além disso, pode-se observar que o melhor material adsorvente é o carvão ativado CA02, confirmando os resultados verificados nas caracterizações de área superficial e mesoporos, os quais apresentaram a maior área superficial e volume de mesoporos, características essenciais para a adsorção de moléculas orgânicas em fases líquidas.
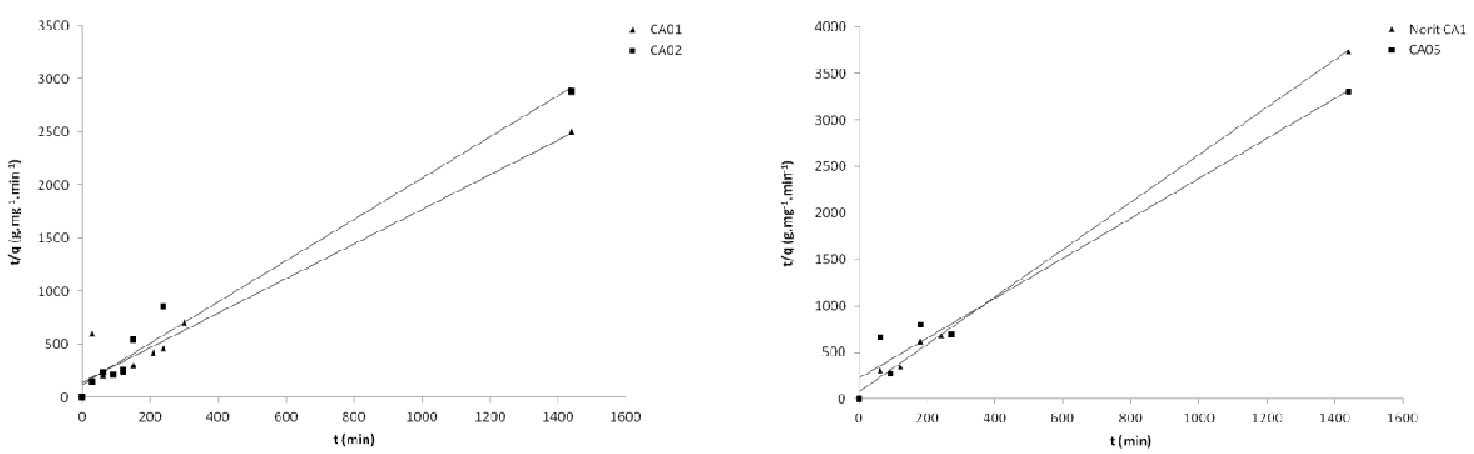

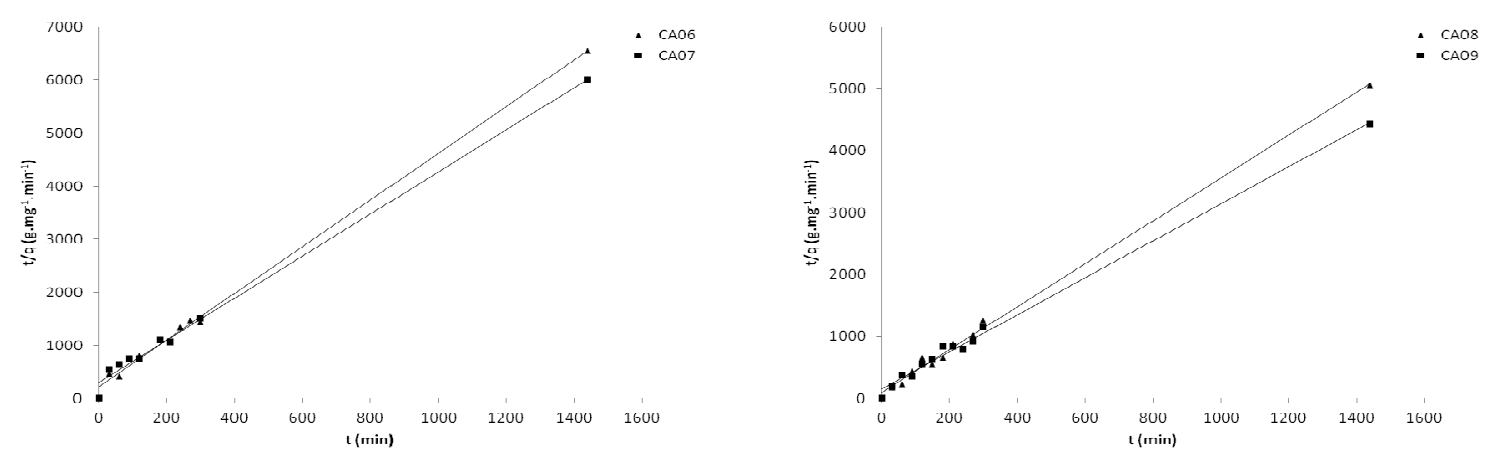

Figura 3 - Linearizações para os modelos cinéticos de pseudossegunda ordem para adsorção de tensoativos aniônicos.

Tabela 3 - Parâmetros cinéticos de pseudossegunda ordem

\begin{tabular}{|c|c|c|c|c|c|c|c|c|}
\hline Amostra & Norit & CA01 & CA02 & CA05 & CA06 & CA07 & CA08 & CA09 \\
\hline $\mathbf{q}_{\mathbf{e}}$ (exp.) $\mathbf{~ m g . g ~}^{-1}$ & 0,385 & 0,500 & 0,575 & 0,435 & 0,220 & 0,240 & 0,285 & 0,325 \\
\hline $\mathbf{q}_{\mathbf{e}}$ (calc.) $\mathbf{~ m g . g}^{-1}$ & 0,393 & 0,514 & 0,613 & 0,466 & 0,227 & 0,252 & 0,288 & 0,325 \\
\hline Desvio (\%) & 2,03 & 2,72 & 6,20 & 6,65 & 3,08 & 4,76 & 0,01 & 2,69 \\
\hline $\mathbf{k}_{\mathbf{2}}$ (g.mg $^{-1} \cdot \mathbf{m i n}^{-1}$ ) & 0,078 & 0,031 & 0,019 & 0,020 & 0,088 & 0,053 & 0,132 & 0,059 \\
\hline $\mathbf{R}^{\mathbf{2}}$ & 0,9978 & 0,9500 & 0,9800 & 0,9697 & 0,9966 & 0,9946 & 0,9969 & 0,9945 \\
\hline
\end{tabular}

Os resultados apresentados para o adsorvente CA02 demonstraram-se similares aos encontrados na literatura, sendo o valor de $\mathrm{k}_{2}\left(0,019 \mathrm{~g} \cdot \mathrm{mg}^{-1} \cdot \mathrm{min}^{-1}\right)$ próximo aos determinados por Taffarel e Rubio (2010) (0,021 g. mg $\left.{ }^{-1} \cdot \mathrm{min}^{-1}\right)$ e Adak et al. (2005) $\left(0,012 \mathrm{~g} \cdot \mathrm{mg}^{-1} \cdot \mathrm{min}^{-1}\right)$. O valor da quantidade adsorvida no equilíbrio pode ser comparada ao resultado de $0,772 \mathrm{mg} \cdot \mathrm{g}^{-1}$ encontrado por Cotoruelo et al. (2009).

As amostras CA03 e CA04, apresentaram ajustes coerentes ao modelo de pseudo-primeira ordem, sendo seus resultados omitidos na Tabela 03 por não haver significado físico.

\subsection{Isoterma de Adsorção}

O procedimento para a determinação das isotermas de adsorção de surfactantes aniônicos foi realizado somente para a amostra CA02, devido esta ter apresentado os melhores resultados nas etapas de caracterização e cinética de adsorção, ou seja, o objetivo nesta etapa foi avaliar este processo para um sistema já otimizado.

O equilíbrio de adsorção de surfactantes aniônicos foi expresso em termos de isotermas de adsorção, sendo linearizado para os modelos de Langmuir e Freundlich. A análise das constantes e seus respectivos coeficientes de determinação, conforme Tabela 4, demonstrou que a isoterma 


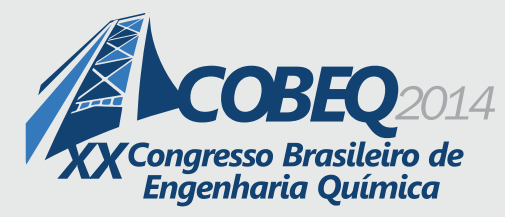

de Freundlich foi o modelo que melhor ajustou-se aos dados experimentais da adsorção do surfactante, ou seja, a adsorção deste composto ocorre por adsorção multicamada em superfície heterogênea.

Tabela 4 - Parâmetros das isotermas de Langmuir e Freundlich

\begin{tabular}{|c|c|c|c|c|c|c|}
\hline \multicolumn{3}{|c|}{ Langmuir } & \multicolumn{4}{|c|}{ Freundlich } \\
\hline$q_{\max }\left(\mathrm{mg} \mathrm{g}^{-1}\right)$ & $\mathrm{K}\left(\mathbf{l . \mathrm { mg } ^ { - 1 } )}\right.$ & $\mathbf{R}^{2}$ & $1 / n$ & $\mathbf{n}$ & $K_{F}\left(\left(m g \cdot g^{-1}\right) \cdot\left(I \cdot g^{-1}\right)^{1 / n}\right)$ & $\mathbf{R}^{2}$ \\
\hline 2,904 & 0,214 & 0,0657 & 0,896 & 1,12 & 0,519 & 0,949 \\
\hline
\end{tabular}

A análise dos dados demonstra que a isoterma de Freundlich é favorável, uma vez que o valor de n está entre 1 e 10, conforme definido anteriormente.

\section{CONCLUSÕES}

$\mathrm{O}$ adsorvente apresentou as melhores características superficiais quando o processo de ativação ocorreu em $600{ }^{\circ} \mathrm{C}$, com uma razão de impregnação de ácido fosfórico e água igual a 1:1 e tempo de ativação de 1 hora. A área superficial, volume de mesoporos e área de mesoporos, para tais parâmetros de ativação foram $663,9 \mathrm{~m}^{2} \cdot \mathrm{g}^{-1}, 0,868 \mathrm{~cm}^{3} \cdot \mathrm{g}^{-1}$ e $373,1 \mathrm{~m}^{2} \cdot \mathrm{g}^{-1}$, respectivamente, superior aos trabalhos relacionados à produção de carvão utilizando cama de aviário, provavelmente devido a expansão da estrutura do material pela ativação química.

Os efeitos de temperatura de ativação e concentração do agente ativador foram avaliadas, sendo possível verificar que o aumento da temperatura em concentrações constantes de ácido aumentou o volume de mesoporos, enquanto o aumento da concentração à $600^{\circ} \mathrm{C}$ ocasionou uma redução em tal volume, o que pode estar ocorrendo devido o excesso de agente ativador causar uma degradação excessiva da estrutura, causando o colapso da mesma.

Os dados cinéticos obtidos demonstram que a amostra CA02 apresentou a maior capacidade de adsorção no equilíbrio, sendo o valor igual a $0,575 \mathrm{mg} \cdot \mathrm{g}^{-1}$, inclusive superior ao carvão comercial Norit CA1.

A isoterma que apresentou o melhor ajuste aos resultados experimentais para os dados de equilíbrio foi a de Freundlich, sendo os resultados obtidos para $K_{F}$ e $n$ iguais a 0,519 ((mg.g $\left.\left.{ }^{1}\right) .\left(1 . \mathrm{mg}^{-1}\right)^{1 / \mathrm{n}}\right)$ e 1,12 , respectivamente.

O conjunto de resultados apresentados neste trabalho indica a cama de aviário como um adsorvente alternativo de baixo custo com grande potencial para remoção de surfactantes aniônicos presentes em corpos d'água e efluentes industriais, sendo a produção de carvão ativado a partir deste precursor um método alternativo para disposição deste resíduo gerado na produção avícola. 


\section{REFERÊNCIAS BIBLIOGRÁFICAS}

ABOULHASSAN, M. A.; SOUABI, S.; YAACOUBI, A.; BAUDU, M. Removal of Surfactant from Industrial Wastewaters by Coagulation Flocculation Process. International Journal of Enviromental Science Technology, v. 3, n. 4, p. 327-332, 2006.

ADAK, A.; BANDYOPADHYAY, M.; PAL, A. Removal of Anionic Surfactant from Wastewater by Alumina: A Case Study. Colloids and Surfaces A: Physicochemical and Engineering Aspects, n. 254, p. 165-171, 2005.

APHA; AWWA; WEF. Standard Methods for the Examination of Water and Wastewater. 21 ed. Washington, EUA, 2005. 1368 p.

ASSOCIAÇÃO BRASILEIRA DE NORMAS TÉCNICAS. NBR 10738: Água - Determinação de surfactantes aniônicos pelo método espectrofotométrico do azul-demetileno. Rio de Janeiro, 1989. $7 \mathrm{p}$.

COTORUELO, L. M.; MARQUÉS, M. D.; MIRASOL, J. R.; RODRÍGUEZ, J. J.; CORDERO, T. Lignin-Based Activated Carbons for Adsorption of Sodium Dodecylbenzene Sulfonate: Equilibrium and Kinetic Studies. Journal of Colloid and Interface Science, v. 332, p. 39-45, 2009.

GAVALA, A.; AHRING, B. K. Inhibition of the Anaerobic Digestion Process by Linear Alkylbenzene Sulfonates. Biodegradation, n. 13, p. 201-209, 2002.

LIMA, I. M.; MCALOON, A.; BOATENG, A. A. Activated Carbon from Broiler Litter: Process Description and Cost of Production. Biomass and Bioenergy, v. 32, p. 568-572, 2008.

MUNGRAY, A. K.; KUMAR, P. Anionic Surfactants in Treated Sewage and Sludges: Risk Assessment to Aquatic and Terrestrial Environments. Bioresource Technology, n. 99, p. 29192929, 2008.

NAHIL, M. A.; WILliAMS, P. T. Pore Characteristics of Activated Carbons from the Phosphoric Acid Chemical Activation of Cotton Stalks. Biomass and Bioenergy, v. 37, p. 142149, 2012.

PINHEIRO FILHO, J. D. Degradação de Alquilbenzeno Linear Sulfonado em Reator Anaeróbio Operado em Bateladas Sequenciais com Biomassa Imobilizada em Carvão Vegetal. São Carlos: Dissertação de Mestrado. Programa de Pós-Graduação em Hidráulica e Saneamento da Escola de Engenharia de São Carlos da Universidade de São Paulo, 2008. 85 p.

SERRANO, V. G.; CORREA, E. M. C.; GONZÁLEZ, M. C. F.; FRANCO, M. F. A.; GARCÍA, A. M. Preparation of Activated Carbons from Chestnut Wood by Phosphoric Acid - Chemical Activation. Study of Microporosity and Fractal Dimension. Materials Letters, v. 59, p. 846-853, 2005.

TAFFAREL, S. R.; RUBIO, J. Adsorption of Sodium Dodecyl Benzene Sulfonate from Aqueous Solution Using a Modified Natural Zeolite with CTAB. Minerals Engineering, v. 23, p. 771-779, 2010. 